\title{
El desarrollo de las ONG de México y su coincidencia con los Objetivos para el Desarrollo Sostenible de Naciones Unidas
}

\section{Laura Rodríguez Cardozo}

RESUMEN: Después de décadas de discusiones y acuerdos internacionales, en 2015 la Organización de las Naciones Unidas adoptó la "Agenda 2030 para el Desarrollo Sostenible" con la finalidad de contribuir a la solución de los actuales problemas de la humanidad. Frente a la complejidad de los mismos, su cumplimiento no puede quedar exclusivamente en manos de los gobiernos, de ahí la importancia del papel a cumplir por parte de la sociedad civil. En ese contexto, el objetivo del presente trabajo es presentar un panorama general del desarrollo de las Organizaciones no Gubernamentales (ONG) en el país que permita analizar su coincidencia con los objetivos de la mencionada Agenda, por medio de análisis documental complementado con información directa proporcionada por actores clave.

El artículo incluye una referencia a los principales conceptos, la evolución del Desarrollo Sustentable, una revisión de enfoques teóricos y estudios sobre las ONG y su contribución al medio ambiente y una descripción general de la situación mexicana en torno del tema: marco legal, registros de ONG disponibles, su número y distribución. Posteriormente se presentan los criterios utilizados para seleccionar el grupo de 21 casos que se analiza en el trabajo. Los anteriores se caracterizan mediante un conjunto de variables que incluyen sus actividades, su antigüedad y experiencia, su relación con el gobierno y la sociedad civil, así como el origen de sus recursos, para finalizar analizando la contribución de sus actividades a los 17 objetivos que conforman la Agenda 2030.

Se concluye que el número de ONG mexicanas ha tenido un rápido crecimiento concentrado en las especialmente vinculadas al Desarrollo Sostenible y en las regiones Sur y Centro del país, que éstas presentan una antigüedad considerable y una amplia diversidad de temas y regiones cubiertas, complementarias de las acciones gubernamentales. En síntesis, la coincidencia de sus actividades con el Desarrollo Sostenible es relevante en el contexto nacional y fundamental en algunas regiones del país con menor presencia de programas gubernamentales. Sin embargo, se llama la atención sobre los riesgos de trabajar exclusivamente en alguno o algunos de los tres ejes del desarrollo sustentable, provocando efectos indeseados en el resto.

PALABRAS CLAVE: Objetivos para el Desarrollo Sostenible, Organizaciones no gubernamentales / Organizaciones de la Sociedad Civil, evaluación, México.

CLAVES ECONLIT: L31, Q01, 013.

Cómo citar este artículo / How to cite this article: RODRÍGUEZ, L. (2017): "El desarrollo de las ONG de México y su coincidencia con los Objetivos para el Desarrollo Sostenible de las Naciones Unidas", CIRIEC-España, Revista de Economía Pública, Social y Cooperativa, 91, 59-84.

Correspondencia: Laura Rodríguez Cardozo. Dirección postal: Rodríguez Saro 424, B 402, Col. Del Valle, CP 03100, Cd. de México, México. Posdoctorante de la Universidad Autónoma Metropolitana, Ciudad de México. Doctora en Medio Ambiente y Desarrollo, Universidad de Baja California. E-mail: rocalaura@gmail.com. 


\section{EXPANDED ABSTRACT}

\section{Development of Mexican Non-Governmental Organizations and its coincidences with the United Nations Sustainable Development Goals}

In 2015, after decades of international agreements, the United Nations adopted the "Agenda 2030 for Sustainable Development", the action plan for the next 15 years to try to solve the current human development problems. This strategy includes 17 goals (no poverty; zero hunger; good health and well being; quality education; gender equality; clean water and sanitation; affordable and clean energy; decent work and economic growth; industry, innovation and infrastructure; reduced inequalities; sustainable cities and communities; responsible consumption and production; climate action; life below water; life on land; peace, justice and strong institutions; and partnerships for the goals), and 169 targets that are integrated together and are non divisible, and which cover environmental, social and economic aspects. To achieve these goals and targets it is necessary that governments and civil society participate together.

México is one of the countries that signed the Agenda 2030. It is characterized by its big extension (1'973,000 km2) and population (123.5 millions), low economic growth of 2.3\% in 2016 (El Economista, 2017), a high poverty level of $43.6 \%$ (Coneval, 2016), and a huge biodiversity (fifth place worldwide according to Llorente-Bousquets y Ocegueda, 2008). Because of this complex situation, it is particularly important to assess the contribution of Non Governmental Organizations (NGOs) to Mexican sustainability. Thus, the objective of the this paper is to propose a general overview of the development of the Mexican NGOs, and to analyse the degree of coincidence of their activities with the UN Agenda 2030's framework for sustainable development.

We use a framework close to constructivism and principally qualitative methods, including analysing and researching documentary information and statistic data, while maintaining an interdisciplinary perspective to study the three fundamental sustainability aspects (environmental, social and economic).

The information sources included mainly NGOs web pages, the Ministry of Government (Segob), the Social Development Institute (Indesol), complemented with direct information provided by different stakeholders from the Ministry of the Environment and Natural Resources (Semarnat) and NGOs', which provide different perspectives. 


\section{EL DESARROLLO DE LAS ONG DE MÉXICO Y SU COINCIDENCIA CON LOS OBJETIVOS PARA EL DESARROLLO SOSTENIBLE DE LAS NACIONES UNIDAS}

We began by identifying the NGOs and their regional localization. Mexican national records have more than 30,000 NGOs (according to Indesol). The NGOs activity in México is regulated by the law called Ley Federal de Fomento a las Actividades Realizadas por Organizaciones de la Sociedad Civil (LFFAROSC), approved in 2004. This law establishes 19 NGOs activities, and activity number 12 relates to sustainable development (SD) and includes: advice for the use of natural resources; environmental, flora and fauna protection; preservation and restoration of the ecological balance; as well as, sustainable development promotion at regional and community levels in urban and rural areas. We work with a subgroup of 5,636 NGOs related to SD, based on activity 12 of LFFAROSC.

Later, we reduce the group to $21 \mathrm{NGOs} \mathrm{recognized} \mathrm{for} \mathrm{their} \mathrm{contribution} \mathrm{at} \mathrm{national} \mathrm{level} \mathrm{and} \mathrm{which}$ have accessible information. These 21 cases where selected by the characterization of a set of variables including their activities, their age and experience, their relationship with the government and the civil society, as well as, the origin of their resources. Then, the contribution from their activities to the 17 Sustainable Development Goals included in the Agenda 2030 is analysed.

It was found that the Mexican NGOs had fast growth and that they have been focused on activity 12 of the LFFAROSC. Also the goals proposed in the Agenda 2030 related to the sustainable cities and communities, life on land, responsible consumption and production, quality education, and affordable and clean energy are covered by the NGOs. In contrast, some of the goals are not the main focus of the analysed NGOs, such as: no poverty, zero hunger, reduced inequalities and quality education (in addition to environmental awareness), among others. Nevertheless, these goals may be covered by NGOs that work in other LFFAROSC activities. Other goals that are not contemplated by the law, particularly those related to economic and political topics (decent work and economic growth; industry, innovation and infrastructure; and peace, justice and strong institutions), where NGOs have less possibility to act. Hence, these responsibilities depended mostly on the government.

In summary, the NGOs contribution to achieve the goals of the Agenda 2030 is relevant in the national context and fundamental in some country regions with less support for governmental programs.

There are some limitations to this study: 1) the 21 NGOs selected for the analysis are not representative of the complete NGOs universe related to SD; 2) the variables used for the analysis where helpful according to the objective of this paper, specialty the description of the NGOs activities, the experience, their relationship with government and civil society, as well as the origin of the resources. Nevertheless, it will be helpful to have data about financial and human resources availability (volunteers and remunerated); and 3) with regards to the production of knowledge of the NGOs, we only did a general description of the variety of documents that they use for the diffusion of knowledge, but a deeper content analysis is necessary to think about quality and utility. 
The work presented constitutes a first approach to the contribution of the main NGOs activities to SD in México. Further research could look at an impact evaluation that identify, quantify, explain and assess the influence to the country in the light of the goals of the UN Agenda 2030.

KEYWORDS: Sustainable Development Goals, Non-Governmental Organizations, Assessment, México. 


\section{EL DESARROLLO DE LAS ONG DE MÉXICO Y SU COINCIDENCIA CON LOS OBJETIVOS PARA EL DESARROLLO SOSTENIBLE DE LAS NACIONES UNIDAS}

\section{Introducción}

El número de ONG ha venido creciendo en toda América Latina, especialmente en las décadas de los setentas y ochentas del siglo pasado (Hurtado, 1995; Thompson y colaboradores, 1995); en el caso mexicano su actividad se relaciona con derechos humanos, salud, alimentación, educación, medio ambiente, etc. (Méndez, 1998). En materia ambiental, los trabajos académicos publicados en torno a sus experiencias son escasos; algunas excepciones se encuentran en Torres (1999), Aguirre (2011), Espinosa-Romero et al. (2014) o en trabajos publicados en colaboración con el gobierno como el de la Comisión Nacional para el Conocimiento y Uso de la Biodiversidad (Conabio), la Comisión Nacional de Áreas Naturales Protegidas (Conanp), The Nature Conservancy (TNC) y Pronatura (2007).

Por otra parte, después de décadas de discusiones y acuerdos internacionales, en septiembre de 2015, la Asamblea General de la Organización para las Naciones Unidas (ONU) adoptó la "Agenda 2030 para el Desarrollo Sostenible", un plan de acción para los próximos 15 años con la finalidad de enfrentar los principales problemas actuales de la humanidad. Al aceptarlo, los 193 Estados miembro se comprometen a su implementación.

En esta nueva estrategia se plantean 17 objetivos y 169 metas de carácter integrado e indivisible que abarcan las esferas económica, social y ambiental. Dichos objetivos se elaboraron durante más de dos años de consultas públicas, interacción con la sociedad civil y negociaciones entre los países. La expectativa es que en estas actividades colaboren no solo los gobiernos sino también las organizaciones no gubernamentales (ONG) e incluso las privadas ${ }^{1}$.

México es uno de los países que ha suscrito la Agenda 2030. Se caracteriza por su gran extensión (1'973,000 km²) y población (123.5 millones de personas, El Economista, 2017), su lento crecimiento económico (2.3\% en 2016, El Economista, 2017), sus elevados niveles de pobreza (43.6\%, Coneval, 2016) y su gran biodiversidad (quinto lugar a nivel mundial, Llorente-Bousquets y Ocegueda, 2008). Debido a la complejidad de esta situación, resulta particularmente relevante analizar la contribución de sus ONG a la sustentabilidad; razón por la que el objetivo de este artículo es presentar un panorama general del desarrollo de las mismas en el país que permita analizar su nivel de coincidencia con la propuesta de desarrollo sostenible en el marco de la Agenda 2030 de la ONU. El trabajo se realizó por medio de análisis documental complementado con información directa proporcionada por los actores clave.

1.- Existe consenso sobre la enorme importancia de la Agenda que está siendo analizada en los más diversos eventos académicos. A título de ejemplos recientes, fue analizada en dos de los siete ejes del XXII Congreso Internacional del CLAD sobre la Reforma del Estado y la Administración Pública (Madrid, España, 14 al 17 de noviembre pasado) y fue el tema de la Conferencia La evaluación frente a los Objetivos de Desarrollo Sostenible: transformando la vida a través de la colaboración global y regional, con énfasis en América Latina y el Caribe (Guanajuato, México, 4 al 8 de diciembre pasado). 


\section{Conceptos relevantes}

En 1987, la Comisión Mundial sobre Medio Ambiente y Desarrollo elaboró un documento para la ONU llamado "Nuestro futuro común", conocido como informe Brundland, en donde se popularizó el término Sustainable Development que ha sido traducido al español como desarrollo sostenible (DS), desarrollo sustentable o desarrollo durable (ONU, 1987).

Para alcanzar el DS es fundamental armonizar tres elementos básicos, el crecimiento económico, la inclusión social y la protección del medio ambiente, los cuales están interrelacionados y son esenciales para el bienestar de las personas y las sociedades (ONU, s/f). Además, se debe tomar en cuenta su sostenibilidad en el tiempo, ya que el DS debe ser capaz de satisfacer las necesidades del presente sin comprometer las propias de las generaciones futuras. Este amplio acuerdo contó desde su inicio con algunas voces que cuestionaban la viabilidad del correcto equilibrio entre estos elementos (Brand y Gorg, 2000).

En relación con el medio ambiente es posible distinguir a quienes lo identifican estrictamente con la naturaleza (medio biofísico) y se refieren al daño que el crecimiento económico provoca sobre ésta, y a quienes lo vinculan a las condiciones de vida de los hombres en su hábitat (Comolet, 1994).

En cuanto a las $O N G$, ha habido mucha ambigüedad conceptual y se han difundido múltiples trabajos desde los organismos internacionales y las propias organizaciones que enfatizan su voluntarismo, disposición a la participación y funcionamiento democrático; pero la literatura académica que reflexiona sobre las mismas (características, tipos, objetivos, evolución, etc.) es mucho más limitada (Deler et al, 1998; Brand y Gorg, 2000; Muñoz, 2014, entre otros). Sin embargo, las definiciones coinciden en que estas organizaciones no pertenecen a las esferas gubernamentales ni persiguen fines de lucro; comparten con el gobierno actividades de prestación de servicios (sociales, educativos, ambientales) pero, a diferencia de éste, no son coercitivas; y si bien son de carácter privado como las empresas, su actividad no está dirigida al mercado y a la obtención de ganancias (Fernandes, 1994; Concha Cantú, 1997; Canto, 2002; Acotto, 2003). En consecuencia, se refieren a una gran diversidad de organizaciones, agrupaciones y asociaciones.

El término ONG fue acuñado en 1945 por la ONU (Artículo 71 del Capítulo X de la Carta de Naciones Unidas), primer organismo internacional que reconoció su papel y aceptó que sus representantes participaran en el Consejo Económico y Social, aunque sin derecho a voto. Desde 1960 también participan en foros de la Organización de las Naciones Unidas para la Alimentación y la Agricultura (FAO, por su sigla en inglés), la Organización Internacional del Trabajo (OIT) y la Organización Mundial de la Salud (OMS). Por su parte, la sociedad civil organizada, poco a poco, ha 


\section{EL DESARROLLO DE LAS ONG DE MÉXICO Y SU COINCIDENCIA CON LOS OBJETIVOS PARA EL DESARROLLO SOSTENIBLE DE LAS NACIONES UNIDAS}

ido sustituyendo el mencionado término de ONG por el de Organizaciones de la Sociedad Civil (OSC), que ha sido retomado por diversas instituciones gubernamentales mexicanas. Por dicha razón, en este trabajo se utilizarán ambos términos, dependiendo del organismo al que se haga referencia.

En México, el área de Desarrollo Político de la Secretaría de Gobernación (Segob) asumió como definición de OSC: "una asociación de ciudadanos que, haciendo uso de recursos simbólicos y materiales, capacidades organizacionales y afinidades emotivas y morales, actúa colectivamente a favor de alguna causa y persigue un interés material o simbólico situándose por fuera del sistema político, y sin seguir la lógica del mercado" (Olvera, 1998: 2 y 2002: 398).

En cambio, el Instituto Nacional de Estadística, Geografía e Informática (INEGI) las reporta entre las Instituciones sin Fines de Lucro y las define en su glosario como: "entidades jurídicas o sociales creadas para producir bienes y servicios, cuyo estatuto jurídico no les permite ser fuente de ingreso, beneficio u otra ganancia financiera para las unidades que las establecen, controlan o financian; están institucionalmente separadas del gobierno, son autónomas y no imponen coercitivamente la participación en ellas". (INEGl: www.beta.inegi.org.mx/app/glosario/default.html?p=csisflm\#letraGlol).

\section{Evolución del Desarrollo Sostenible}

Como ya se mencionó, el término de Desarrollo Sustentable se introdujo en 1987 por el informe Brundtland. Sin embargo, la preocupación por los problemas ambientales ya se había venido planteando en diversos eventos internacionales como:

- La creación de la Unión Internacional para la Conservación de la Naturaleza en 1948, primera organización que buscaba preservar los recursos naturales.

- La Primera Conferencia Mundial de Parques Nacionales en 1962, que influyó en la declaración de Áreas Naturales Protegidas (ANP) en Norteamérica y México.

- El Programa Hombre y Biósfera de la Organización de las Naciones Unidas para la Educación, la Ciencia y la Cultura (UNESCO, por su sigla en inglés) en 1971, que dio surgimiento a las Reservas de la Biósfera.

- La Conferencia sobre Medio Humano de las Naciones Unidas de 1972 expresa por primera vez la preocupación por el problema ambiental a nivel mundial.

- La creación del Programa de las Naciones Unidas para el Medio Ambiente (PNUMA) de 1972 como herramienta para conservación de especies y ecosistemas. 
A partir de 1987, muchas reuniones internacionales han retomado el tema del Desarrollo Sostenible; se mencionan algunas de las más importantes:

- En 1992 la Cumbre de la Tierra de Río de Janeiro refuerza la idea de "tres pilares" que deben conciliarse en una perspectiva de desarrollo sustentable: el progreso económico, la justicia social y la preservación del medio ambiente.

- Al siguiente año, se aprueba el V Programa de Acción en Materia de Medio Ambiente de la Unión Europea bajo el título "Hacia un desarrollo sostenible", en que se presenta la nueva estrategia comunitaria en materia de medio ambiente y las acciones planeadas para 19922000.

- En 1994 se produce la Primera Conferencia de Ciudades Europeas Sostenibles en Aalborg (Dinamarca).

- Iniciado el nuevo siglo (2000), se definen nuevos objetivos y metas dentro de la Cumbre del Milenio de las Naciones Unidas, que incluye el tema de la degradación del ambiente.

- Al año siguiente se define el VI Programa de Acción en Materia de Medio Ambiente de la Unión Europea. "Medio ambiente 2010: el futuro en nuestras manos", que define las prioridades y objetivos de la política medioambiental de la Comunidad Europea para contribuir a la estrategia de desarrollo sostenible (http://europa.eu/legislation_summaries/agriculture/environment//28027_es.htm).

- En 2002 se reúne en Johannesburgo la Conferencia Mundial sobre Desarrollo Sostenible, donde se reafirmó el papel del desarrollo sostenible para la lucha contra la pobreza y la protección del medio ambiente en la Agenda Internacional, comprometiéndose las partes a reducir el ritmo de pérdida de la biodiversidad (http://www.un.org/spanish/conferences/wssd).

- En 2005 entra en vigor el Protocolo de Kioto sobre la reducción de las emisiones de gases de efecto invernadero, aprobado en 1997.

- Se produce la comunicación de 2006 al Consejo y al Parlamento Europeo sobre una Estrategia temática para el medio ambiente urbano, como parte del VI Programa de Acción en materia de Medio Ambiente de la Unión Europea.

- En 2012 se produce la Conferencia Río+20, que ratificó los acuerdos de protección de la biodiversidad y de pago de servicios ambientales.

- En 2015 se concretó la Agenda 2030 para el Desarrollo Sostenible con sus 17 objetivos que reemplazan los objetivos del Milenio del año 2000: fin de la pobreza; hambre cero; salud y bienestar; educación de calidad; igualdad de género; agua limpia y saneamiento; energía asequible y no contaminante; trabajo decente y crecimiento económico; industria, innovación e infraestructura; reducción de las desigualdades; ciudades y comunidades sostenibles; producción y consumo responsables; acción por el clima; vida submarina; vida de ecosistemas terrestres; paz, justicia e instituciones sólidas; y alianzas para lograr los objetivos. 


\section{EL DESARROLLO DE LAS ONG DE MÉXICO Y SU COINCIDENCIA CON LOS OBJETIVOS PARA EL DESARROLLO SOSTENIBLE DE LAS NACIONES UNIDAS}

A partir de los años setenta, en este contexto internacional, el gobierno mexicano ha recogido el paradigma de la sustentabilidad para integrarlo en la estrategia de conservación. Pueden citarse como eventos relevantes:

- La publicación de la Ley General del Equilibrio Ecológico y la Protección al Ambiente (LGEEPA) en 1988.

- La creación de la Secretaría de Medio Ambiente, Recursos Naturales y Pesca (Semarnap) en 1994.

- La aplicación de los Programas de Desarrollo Regional Sustentable (Proders) desde 1996.

- La creación de la Comisión Nacional de Áreas Naturales Protegidas (Conanp) en el año 2000.

- En 2004, la inclusión en la misión de la Comisión previa del compromiso de fomentar una cultura de la conservación y el desarrollo sustentable de las comunidades asentadas en su entorno.

- La transformación de los Proders en Programas de Conservación para el Desarrollo Sostenible (Procodes) a partir de 2007.

- La introducción en 2008 de la Estrategia Nacional para un Desarrollo Sustentable del Turismo y la Recreación en las Áreas Protegidas de México.

Es en este marco internacional y nacional, en que no se visualiza la contribución de las ONG al desarrollo sostenible y falta información sistematizada al respecto, que se propone un primer acercamiento que permita dimensionar su aporte actual y potencial.

\section{Estudios sobre ONG}

La mayor parte de los estudios sobre estas organizaciones analizan sus recursos, organización, funcionamiento, participación social, relaciones con los gobiernos y resultados (Drucker, 1990; Fernandes, 1994; Fowler et al., 1995; New Economics Foundation, 1998; Canto, 2002; etc.). Muchos están referidos a sus campos de trabajo más frecuentes, ya mencionados: derechos humanos, educación, alimentación, salud, papel de la mujer y de los jóvenes, etc. (González, 1999; López e Hincapié, 2015; Caicedo y Solarte, 2015; etc.). Otros pueden incluir ONG preocupadas por el medio ambiente, como las mencionada en la introducción; pero, al menos en el contexto latinoamericano, es difícil encontrar trabajos que recuperen el enfoque tripartito de la sustentabilidad y la educación ambiental correspondiente (Bermúdez y Lombana, 2009). 
Una revisión más detallada de la literatura al respecto muestra que durante la segunda parte del siglo XX aumenta la constitución y participación de las ONG en el espacio público, medida a través de los recursos financieros manejados, el empleo ofrecido y la atención pública (Hulme y Edwards, 1997). En los años noventa, en que se consolida el enfoque del DS, su presencia ya era significativa y reconocida en los informes del Banco Mundial y en el Plan Nacional de Desarrollo de México 19952000.

Sin embargo, como ya se mencionó, las ONG constituyen un grupo muy heterogéneo. Léna (1998) afirma que, en el caso de Brasil, las fronteras entre diferentes tipos de organizaciones han sido muy laxas, reconociendo: las más antiguas de carácter caritativo y filantrópico, las de apoyo a movimientos populares, y las llegadas al final, en defensa de la ecología, que hacia el fin de siglo pasado fueron las que más crecieron numéricamente, a pesar de enfrentar fuertes fricciones en su contexto. Considera que la etiqueta de ONG convino políticamente a todas en la competencia por acceder a recursos extranjeros y gubernamentales. En ese sentido, la creación de la Asociación Brasilera de ONG (ABONG) en 1991 constituyó un paso significativo en su reconocimiento y creó un nuevo mercado de empleo remunerado alternativo.

Brand y Gorg (2000) proponen diversos aspectos a considerar para la diferenciación de las ONG: la dimensión y estructura de las organizaciones, su posición ideológica y motivación política, su composición (miembros) y relaciones (donadores), su posición frente al Estado (conflictiva, cooptada 0 cooperativa), las empresas privadas y los organismos internacionales, fuentes de financiamiento, niveles de actuación (local, nacional, internacional), y su posición geográfica en el hemisferio Norte o Sur. Esto mostraría ONG con intereses diferentes y hasta contradictorios, que llevan incluso a los autores a cuestionarse si algunas deberían ser consideradas en este conjunto. Lo que ejemplifican en el caso de la International Union for Conservation of Nature (UICN) debido a que fue fundada por el gobierno francés, cumple tareas oficiales, tiene más 60 naciones miembros, financia e implementa proyectos, y por su dimensión, estructura y objetivos debería considerarse un organismo internacional. En el mismo sentido, Pouyllan y Saavedra (1998) destacan la conveniencia de diferenciar dentro del conjunto de ONG a las más importantes, que se identifican como "multinacionales"; mientras Léna (1998) destaca que las grandes ONG ecologistas del Norte tienen suficiente poder para influir incluso en la política del Banco Mundial.

Brand y Gorg también distinguen las ONG para el desarrollo (ONGDs) que financian e implementan proyectos y generalmente están ubicadas en el hemisferio Norte, de las ONG medioambientales que participan en la definición de problemas ecológicos, la creación de conciencia sobre ellos o la definición de políticas públicas, tanto a nivel nacional como internacional (Greenpeace, World Wildlife Fundation for Nature). Entre las primeras, afirman que los aspectos medioambientales se consideran sólo marginalmente, mientras en las segundas, constituyen la orientación dominante. Debido al impulso a la participación social incluido en el capítulo 27 de la Agenda 21 de la ONU, muchas ONG participaron en el debate sobre el DS, algunas con fuerte liderazgo como la IUCN. Se supone que las ONG medioambientales defienden los intereses de la humanidad, pero estos autores plantean que no 


\section{EL DESARROLLO DE LAS ONG DE MÉXICO Y SU COINCIDENCIA CON LOS OBJETIVOS PARA EL DESARROLLO SOSTENIBLE DE LAS NACIONES UNIDAS}

todas actúan de igual forma, sino que utilizan tres variantes diferentes: la retórica que usa el término sin profundizar en su significado, la que busca manejar los problemas a través de la regulación internacional, y la que persigue la creación y fortalecimiento de políticas regionales y locales alternativas desde su base social. Pouyllan y Saavedra (1998) también señalan que los objetivos y acciones de las ONGs más pequeñas suelen extenderse, no sin contradicciones ideológicas o identitarias, más allá de lo meramente ambiental, incluyendo en los casos más comprometidos la defensa de las clases sociales más marginadas. Así el debate de la "economía contra la naturaleza" puede responder a un enfoque marxista, uno neoclásico o a la llamada tendencia "verde".

Aún menos frecuentes que los anteriores son los trabajos que proponen y analizan metodologías para valorar la contribución de las ONG al sector en que trabajan² por varias razones: la propia resistencia de las ONG ante la exigencia de los organismos donantes, el poco valor que le han atribuido a los resultados de la evaluación (que ha venido cambiando en los últimos años), la falta de métodos diseñados ad hoc para valorar su desempeño, entre otros.

Originalmente las ONG actuaban como grupos de presión sobre el gobierno para que incluyera determinados asuntos en su agenda y ofreciera soluciones, pero muchas se han ido transformando en interlocutoras o incluso colaboradoras del gobierno. Como se verá a continuación, esta diversidad de posiciones ha llevado a que, aun cuando se han realizado evaluaciones, en varios estudios se cuestione la contundencia de sus hallazgos y en algunos incluso se sostenga que provocan efectos negativos.

Riddell et al (1997) realizaron una de las investigaciones más completas, cubriendo 26 países. Encontraron insuficiencia de datos fiables, muy poco consenso sobre los métodos de evaluación más apropiados, dificultades para evaluar aspectos como la construcción y el fortalecimiento de la capacidad institucional cuya incidencia y popularidad ha aumentado y, en general, poca claridad sobre los impactos alcanzados en todos los países incluidos.

Entre los 44 trabajos provenientes de muy diversos contextos compilados por Deler et al (1998) para examinar la relación de las ONG con el desarrollo económico, social y político, resaltan dos por su vinculación con el medio ambiente y el desarrollo sostenible en países latinoamericanos: Colombia, Ecuador y Venezuela (Pouyllan y Saavedra) y Brasil (Léna). En el primer caso, los autores aplican la expresión "realismo mágico" para referirse a las excesivas relaciones que mantienen las ONG con el Estado y otros actores en el campo ambiental; mientras en Brasil se refiere una asociación por conveniencia mutua de las organizaciones sociales y ecologistas, que distorsiona los objetivos de cada tipo, provocando ambigüedades, contradicciones y efectos perversos, al punto de cuestionarse si se trata de una "refeudalización de la asistencia a los pobres".

En el mismo tenor, Davies (2001) plantea su preocupación por la limitada capacidad de las ONG para evidenciar y comunicar sus logros. Señala que, a pesar que los organismos bilaterales han financiado estudios de gran escala como el de Riddell et al, que algunas ONG han producido sus propias

2.- Reino Unido es una excepción. 
guías (Roche, 2000), hacen su evaluaciones, síntesis y metaevaluaciones y han publicado algunos libros (Fowler, 1997), poco se sabe sobre los resultados alcanzados según diversos estudios, como el ya citado de Riddell et al o el de Oakley (1999) en los proyectos de 45 ONG danesas en cuatro países. Al estudiar las causas posibles encuentra: a) expectativas demasiado ambiciosas en los Términos de Referencia, con muchos criterios y objetivos del Marco Lógico, que contrastan con conclusiones ambiguas; $b$ ) complejidad debida a las diferentes escalas (internacionales, nacionales, regionales) de actuación de las ONG que deben integrar en su evaluación; c) diversidad de actividades, las que propone simplificar mediante la realización de estudios temáticos o por sectores; d) objetivos vagos relativos a actividades de capacitación, fortalecimiento institucional o desarrollo de la sociedad civil, difíciles de medir pero verificables; e) herramientas utilizadas, como los Marcos Lógicos, más adecuados para la planeación, o los métodos participativos que generan mucha información a analizar; y f) ausencia de líneas basales y adecuados sistemas de monitoreo debido a que se da seguimiento a indicadores de corto plazo y se descuidan los impactos más tardados.

En España, Serrano (2002) se pregunta si la supuesta mayor eficacia de la gestión de las ONG frente al Estado y la empresa privada o su mayor proximidad a la población beneficiaria son o no reales e identifica las dificultades de esta evaluación general, ya que depende de cómo midan sus resultados y los indicadores que usen. Explica esta ambigüedad por el carácter disperso y no coordinado de las numerosas ONG, así como la multiplicidad de proyectos que cada una maneja, y señala que los estudios de caso realizados apuntan a que dichas ventajas son inexistentes. Estos resultados cuestionan el valor añadido que aporta su ejecución de proyectos.

En América Latina, en el año 2000, la ABONG tradujo y adaptó el libro publicado por Roche (1999) sobre la evaluación de impacto del trabajo de las ONG ante la ausencia de publicaciones propias. México también se ha caracterizado por contar con muy pocos trabajos de evaluación de sus ONG. Entre 1991 y 2003, existió una consultora (Espiral Consultora S.C.) que brindaba asesorías especializadas a ONG, incluyendo algunos trabajos de evaluación. Otros ejemplos se han realizado para informar a las organizaciones que patrocinan sus proyectos (Novib, The William and Flora Hewlett Foundation, etc.). Estos esfuerzos se vinculaban a la evaluación de objetivos sociales, pero la contribución de las ONG a la compleja mezcla de objetivos presentes en el DS constituye una tarea casi sin antecedentes. En este caso se han diseñado conjuntos de indicadores que cubren sus tres aspectos para el caso de Áreas Naturales Protegidas (Hockings et al., 2000; Pomeroy, 2,006; Leverington, 2008), los que han sido modificados para dar mayor relevancia a los temas sociales propios de México (Rodríguez, 2015), pero no han sido aplicados en ONG. Recientemente, Castañeda (2016) analizó la contribución de cinco ONG de Quintana Roo en un proyecto de DS impulsado por Conabio en el Corredor Biológico Mesoamericano.

En síntesis, se trata de un tema poco trabajado a nivel académico, especialmente en el contexto latinoamericano, y con apreciaciones divergentes sobre el papel que juegan o podrían jugar las ONG en el marco de una política de DS. 


\section{Métodos}

El presente trabajo se propuso cubrir la primera etapa de un proceso de evaluación, destinada a conocer las actividades de las ONG mexicanas (en su mayoría preexistentes a la Agenda 2030 y creadas con objetivos diversos) para valorar su nivel de coincidencia con los ODS, mediante un enfoque cercano al constructivismo. Ante la insuficiente información sistematizada sobre el trabajo de las ONG en México fue necesario empezar por identificar al conjunto y su localización regional. En la medida en que los registros nacionales mencionan un universo de más de 30,000 ONG (Instituto Nacional de Desarrollo Social, Indesol), se seleccionaron primeramente las vinculadas con el DS $(5,636)$ $y$, en una segunda etapa, un subconjunto de tamaño manejable en el trabajo, constituido por las ONG de DS más reconocidas por su contribución a nivel nacional y cuya información resultó accesible. En consecuencia, la muestra lograda no es representativa del universo total pero incluye a más del $77 \%$ de las ONG destacadas por la Secretaría de Medio Ambiente y Recursos Naturales (Semarnat) debido a su contribución al DS.

Las fuentes de información incluyeron principalmente las páginas web de las propias ONG, la Segob, el Indesol y la Semarnat; complementariamente, se obtuvieron datos adicionales mediante consulta por Internet a las mismas organizaciones para contar con los puntos de vista de los diferentes actores involucrados.

Los métodos fueron predominantemente cualitativos incluyendo el análisis de documentos y datos estadísticos, desde una perspectiva interdisciplinaria capaz de estudiar los tres ejes de la sustentabilidad. La información recogida se sistematizó en torno a algunas de las características señaladas por Brand y Gorg (2000) y otras pertinentes para el estudio. Finalmente, se utilizó el análisis comparativo para contrastar las actividades de las ONG con los ODS.

\section{Resultados}

\subsection{ONG/OSC de México: marco legal y registros}

En México, las OSC están reconocidas en el Código Civil Federal y su estatus legal es variable. Según el Registro Federal de OSC, en julio de 2015, predominaban ampliamente las Asociaciones 
Civiles (28,644), pero también se podían encontrar algunas Instituciones de Asistencia Privada (984), Sociedades Civiles (558), Asociaciones de Beneficencia Privada (108), Instituciones de Beneficencia Pública (46) y otras (39). La diferencia entre Sociedades Civiles y Asociaciones Civiles es que las primeras realizan un fin común lícito, preponderantemente económico, y las asociaciones civiles realizan un fin común preponderantemente no económico, es decir, un fin deportivo, religioso, cultural, etc. El $96 \%$ de las OSC está constituido bajo la figura pública de asociación civil.

La actividad de las OSC está regulada por la Ley Federal de Fomento a las Actividades Realizadas por Organizaciones de la Sociedad Civil (LFFAROSC) aprobada en 2004, pero también existen otras específicas en cada entidad federativa. Esta Ley federal define a las OSC como organizaciones legalmente constituidas, sin fines de lucro y de ayuda a terceros, que no persiguen objetivos políticos, partidistas ni religiosos. Su artículo 5, capítulo II, establece las 19 actividades sociales y ambientales de las OSC que son objeto de fomento, incluyendo entre ellas la No. 12, de especial interés para este trabajo: apoyo al aprovechamiento de los recursos naturales, la protección del ambiente, la flora y la fauna, la preservación y restauración del equilibrio ecológico, así como la promoción del desarrollo sustentable a nivel regional y comunitario, de las zonas urbanas y rurales. El $17.8 \%$ del total de las OSC $(5,636)$ reportan esta actividad (Sistema de Información del Registro Federal de las OSC, s/f).

La LFFAROSC creó el Registro Federal de las Organizaciones de la Sociedad Civil a cargo del Indesol. El mismo cumple, entre otros, con el objetivo de difundir las actividades y el impacto de aquellas ONG que cumplen con los requisitos establecidos en la misma norma, como estar formalmente constituidas, y les otorga una clave única (CLUNI). Las OSC inscritas adquieren derechos y obligaciones, entre los que resaltan: acceder a los apoyos y estímulos públicos, ser respetadas en la toma de decisiones relativa a sus asuntos internos, colaborar como instancias de participación y consulta, participar en los mecanismos de contraloría social, gozar de incentivos fiscales, recibir donativos y aportaciones, informar anualmente sobre los apoyos y estímulos públicos federales recibidos y el balance de su situación patrimonial, contable y financiera, notificar las modificaciones a la información entregada y su disolución. Entre 2010 y 2013 el número de apoyos recibidos por las OSC de parte de Indesol se quintuplicó, mientras el número de éstas sólo se triplicó, alcanzando en promedio cerca de dos apoyos anuales por OSC. Sin embargo, los montos otorgados tendieron a reducirse drásticamente (pasaron en promedio de aproximadamente 83,000 a 43,000 dólares entre 2010 y 2013). Las principales acciones financiadas que reportaron logros fueron la creación de nuevos programas, la reorientación de la política y la modificación de programas existentes (Sedesol/Indesol, 2015).

La Segob también cuenta con un directorio de las organizaciones que voluntariamente solicitan estar registradas con fines meramente informativos (darse a conocer), pero éstas pueden no estar constituidas legalmente ante Notario. Se trata de un registro que incluye un número muy elevado de organizaciones de los más diversos tipos, clasificadas sólo por entidades federativas, lo que no permite ordenarlas en función de su actividad (Segob, s/f). 


\section{EL DESARROLLO DE LAS ONG DE MÉXICO Y SU COINCIDENCIA CON LOS OBJETIVOS PARA EL DESARROLLO SOSTENIBLE DE LAS NACIONES UNIDAS}

\subsection{ONG: número y distribución por entidades federativas, regiones y actividades}

El número de OSC ha crecido sensiblemente después del sismo de 1985, destacando los años 2013 y 2014 con 4,677 y 4,641 nuevas inscripciones, para alcanzar un total actual superior a 30,000 (Ver Cuadro No.1). Se estima que más del 70\% están actualmente activas (Registro del Indesol). Es difícil hacer comparaciones numéricas con otros países por la imprecisión de los datos, pero México se encuentra aún lejos de naciones como Brasil o Argentina que reportan alrededor de 350,000 y 100,000 OSC respectivamente (http://lanic.utexas.edu/la/region/ngos/indexesp.html).

La distribución de las OSC por entidades federativas es muy desigual; en julio de 2015 la mayoría se localizaba en el entonces Distrito Federal, hoy Ciudad de México $(6,113)$, seguida del Estado de México, Veracruz, Oaxaca y Chiapas. Agrupadas por regiones, las más numerosas corresponden a las regiones Centro y Sur.

Sin embargo, el número de OSC por entidades está determinado en parte por el tamaño de la población que allí habita, por lo que es conveniente analizar su densidad asociativa (OSC por 100,000 habitantes), como aparece en la quinta columna del cuadro mencionado. La mayor concentración se localiza en la capital (69.0), coincidiendo con el mayor número de OSC, pero le siguen los estados de Durango, Oaxaca, Morelos, Colima, etc. Nuevamente, agrupando las entidades por regiones, la mayor concentración se localiza en la región Centro, seguida de la región Sur.

El número total de OSC se concentra en la actividad No. 12 (relativa al DS) en la mayoría de las entidades y en todas las regiones, resaltando la región Sur (30\% del total), la Centro $(18.65 \%)$ y la Sureste (17.3\%). Su distribución es muy similar a la general: siendo mayor en el DF, seguido de Oaxaca, Veracruz y el Estado de México y concentrándose en las regiones Centro y Sur. En materia de densidad (séptima columna del Cuadro No. 1) el orden se modifica y los primeros lugares corresponden a las entidades de Oaxaca, Distrito Federal, Chiapas y Durango, y a las regiones Sur y Centro.

Resaltan las regiones Noreste y Occidente por su menor densidad de OSC en general y en la actividad 12 en particular, aunque seguidas muy de cerca por la región Noroeste. A nivel estatal la menor densidad de OCS en general se encuentra en Nuevo León y Tamaulipas, mientras que con respecto a la actividad 12 la misma se localiza en Nuevo León y Jalisco.

\subsection{ONG incluidas en el estudio}

Dentro del universo de 5,636 (ver Cuadro No.1) OSC identificadas con actividad 12 (DS), se realizó una búsqueda electrónica para identificar las que estaban vigentes y mostraban impacto a nivel nacional. Con ese listado, se consultó la opinión de la Semarnat ${ }^{3}$ en cuanto a su colaboración y contribución a

3.- Información brindada por su Dirección General Adjunta de Participación Ciudadana de la Unidad Coordinadora de Participación Social y Transparencia (UCPAST). Junio de 2016. 
la sustentabilidad. Después de esta depuración se obtuvo información en las páginas web de las ONG preseleccionadas y se las contactó vía electrónica para solicitarles datos complementarios ${ }^{4}$.

\section{Cuadro 1. Información de OSC por entidades federativas y regiones 2015}

\begin{tabular}{|c|c|c|c|c|c|c|}
\hline Región & $\begin{array}{l}\text { Entidades } \\
\text { federativas }\end{array}$ & $\begin{array}{l}\text { Población } \\
\text { por entidad }\end{array}$ & $\begin{array}{l}\text { Número } \\
\text { de OSC }\end{array}$ & $\begin{array}{c}\text { OSC por } 100,000 \\
\text { habitantes }\end{array}$ & $\begin{array}{c}\mathrm{N}^{\circ} \text { de OSC } \\
\text { Actividad } 12\end{array}$ & $\begin{array}{l}\text { OSC de actividad } 12 \\
\text { por } 100,000 \text { hab. }\end{array}$ \\
\hline 1.- Región & Baja California & $3 ’ 484,150$ & 1,055 & 30.3 & 95 & 2.7 \\
\hline \multirow[t]{3}{*}{ Noroeste } & Baja California Sur & 763,929 & 191 & 25.0 & 46 & 6.0 \\
\hline & Sinaloa & 2'984,571 & 460 & 15.4 & 58 & 1.9 \\
\hline & Sonora. & 2'932,821 & 558 & 19.0 & 71 & 2.4 \\
\hline Subtotales/promedio & & $10^{\prime} 165,471$ & 2,264 & 22.2 & 270 & 2.7 \\
\hline 2.-Región & Coahuila & $2^{\prime} 960,681$ & 739 & 25.0 & 75 & 2.5 \\
\hline \multirow[t]{6}{*}{ Noreste } & Chihuahua & 3710,129 & 907 & 24.5 & 74 & 2.0 \\
\hline & Durango & $1 ' 764,726$ & 868 & 49.2 & 143 & 8.1 \\
\hline & Nuevo León & $5^{\prime} 085,848$ & 654 & 12.9 & 48 & 0.9 \\
\hline & San Luis Potosí & $2 ' 753,478$ & 383 & 13.9 & 52 & 1.9 \\
\hline & Tamaulipas & $3^{\prime} 543,366$ & 366 & 10.3 & 53 & 1.5 \\
\hline & Zacatecas & $1^{\prime} 576,078$ & 351 & 22.3 & 65 & 4.1 \\
\hline Subtotales/promedio & & $21 ' 394,306$ & 4,268 & 19.9 & 510 & 2.4 \\
\hline 3.-Región & Aquascalientes & 1'287,660 & 354 & 27.5 & 32 & 2.5 \\
\hline \multirow{6}{*}{ Occidente } & Colima & 723,455 & 257 & 35.5 & 35 & 4.8 \\
\hline & Guanajuato & $5^{\prime} 817,614$ & 854 & 14.7 & 107 & 1.8 \\
\hline & Jalisco & 7'931,267 & 1,189 & 15.0 & 101 & 1.3 \\
\hline & Michoacán & 4'596,499 & 1,165 & 25.3 & 188 & 4.1 \\
\hline & Nayarit & $1 ' 223,797$ & 318 & 26.0 & 47 & 3.8 \\
\hline & Querétaro & $2^{\prime} 004,472$ & 480 & 24.0 & 72 & 3.6 \\
\hline Subtotales/promedio & & $23^{\prime} 584,764$ & 4,617 & 19.6 & 582 & 2.5 \\
\hline \multirow[t]{6}{*}{ 4.-Región Centro } & Distrito Federal & $8^{\prime} 854,600$ & 6,113 & 69.0 & 1002 & 11.3 \\
\hline & Estado de México & $16^{\prime} 870,388$ & 2,626 & 15.6 & 512 & 3.0 \\
\hline & Hidalgo & $2^{\prime} 878,369$ & 638 & 22.2 & 145 & 5.0 \\
\hline & Morelos & $1 ' 920,350$ & 770 & 40.1 & 152 & 7.9 \\
\hline & Puebla & $6^{\prime} 193,836$ & 1,130 & 18.2 & 273 & 4.4 \\
\hline & Tlaxcala & $1 ' 278,308$ & 417 & 32.6 & 84 & 6.6 \\
\hline Subtotales/promedio & & $37 ' 995,851$ & 11,694 & 30.8 & 2,168 & 5.7 \\
\hline \multirow[t]{4}{*}{ 5.-Región Sur } & Chiapas & $5,252,808$ & 1,313 & 25.0 & 459 & 8.7 \\
\hline & Guerrero & $3,568,139$ & 654 & 18.3 & 180 & 5.0 \\
\hline & Oaxaca & 4'012,295 & 1,921 & 47.9 & 697 & 17.4 \\
\hline & Veracruz & $8^{\prime} 046,828$ & 2,171 & 27.0 & 514 & 6.4 \\
\hline Subtotales/promedio & & $20^{\prime} 880,070$ & 6,059 & 29.0 & 1,850 & 8.9 \\
\hline \multirow[t]{4}{*}{ 6.-Región Sureste } & Campeche & 907,878 & 202 & 22.3 & 34 & 3.7 \\
\hline & Quintana Roo & $1^{\prime} 574,824$ & 350 & 22.2 & 64 & 4.1 \\
\hline & Tabasco & $2,383,900$ & 426 & 17.9 & 77 & 3.2 \\
\hline & Yucatán & $2 ' 118,762$ & 499 & 23.6 & 81 & 3.8 \\
\hline Subtotales/promedio & & 6'985,364 & 1,477 & 21.1 & 256 & 3.7 \\
\hline Totales & & $121^{\prime} 005,826$ & 30,379 & & 5,636 & \\
\hline
\end{tabular}

FUENTE: Elaboración propia con datos de julio de 2015 del Sistema de Información del Registro Federal de las OSC (SIRFOSC) y estimaciones de población de INEGI a mediados de 2015. 


\section{EL DESARROLLO DE LAS ONG DE MÉXICO Y SU COINCIDENCIA CON LOS OBJETIVOS PARA EL DESARROLLO SOSTENIBLE DE LAS NACIONES UNIDAS}

Nuestro grupo de estudio quedó constituido por ocho de las 12 OSC identificadas por Semarnat ( $77 \%$ del total considerado relevante). El 23\% restante tuvo que descartarse debido a no contar con suficiente información para realizar su estudio completo, si bien dos OSC de este subgrupo contestaron una pregunta sobre sus principales aportes. Además, se incluyeron otras ocho OSC, en función de su prestigio local y la información disponible, que completan el grupo de estudio de 21 organizaciones ${ }^{5}$.

\subsection{Caracterización de las OSC seleccionadas}

Para la caracterización de las OSC se consideraron los aspectos propuestos por Brand y Gorg (2000) de los que se dispuso de información: origen del financiamiento, tipo de vinculación con los niveles de gobierno y nivel de actuación. Sin embargo, se agregaron otras características consideradas pertinentes para el estudio como: tipo de ecosistema en el que trabajan, origen, alcance de sus acciones, antigüedad, entre otras.

La mayoría de las OSC analizadas son de carácter nacional o sedes nacionales de organizaciones internacionales (TNC; Fondo Mundial para la naturaleza, WWF por su siglas en inglés; Greenpeace) y trabajan en múltiples regiones del territorio nacional, aunque algunas se especializan en regiones más específicas relacionadas con su actividad (Comunidad y Biodiversidad en mares y costas, Grupo de Ecología y Conservación de Islas en estas últimas, Grupo de Estudios Ambientales AC en el campo).

De igual manera, son diversos los ecosistemas que atienden, con excepción de unos pocos casos especializados (Comunidad y Biodiversidad, Consejo Civil Mexicano para la Silvicultura Sostenible, Institute for Transportation \& Development Policy, Grupo de Ecología y Conservación de Islas, Grupo de Estudios Ambientales AC). Ellos desempeñan actividades muy variadas entre las que destacan la conservación, la cultura y educación ambiental, la restauración, la protección, el manejo y recuperación de especies en peligro de extinción, el aprovechamiento racional y sustentable, entre otras. También se mencionan recurrentemente temas más amplios como la gestión y el desarrollo de políticas públicas. Además, hay ejemplos que promueven asuntos menos compartidos como el derecho ambiental (CEMDA y Acción Cultural Madre Tierra AC), las herramientas legales (DAN) y otros dedicados a temas más concretos como: cambio climático, ANP, bosques, cuencas, mares, energía, clima, agua, alimentos, gestión de residuos, transporte y sustentabilidad en la producción rural y pesquera.

4.- Información recogida en agosto de 2016.

5.- Centro Mexicano de Derecho Ambiental (CEMDA); 2) Pronatura Noroeste; 3) Espacios Naturales y Desarrollo Sustentable; 4) Fondo Mexicano para la Conservación de la Naturaleza; 5) Beta Diversidad; 6) Naturalia; 7) World Wildlife Fund México (WWF); 8) Conservación Internacional; 9) Greenpeace México; 10) Comunidad y Biodiversidad; 11) The Nature Conservancy (TNC); 12) Consejo civil Mexicano para la Silvicultura Sostenible; 13) Institute fot Transportation \& Development Policy; 14) Acción Cultural Madre Tierra e Iniciativa Mexicana de Aprendizaje para la Conservación; 15) ICLEI- Gobiernos Locales por la Sustentabilidad; 16) Biocenosis; 17) Grupo de Ecología y Conservación de Islas (GECI); 18) Grupo de Estudios Ambientales; 19) Asociación Nacional de Energía Solar; 20) Fondo para la Comunicación y la Educación Ambiental; y 21) Estudios Rurales y Asesoría Campesina. 
El inicio de su trabajo en la región varía entre 1977 (Grupo de Estudios Ambientales AC) y 2005 (Beta Diversidad), con un promedio elevado de antigüedad (24 años). Algunas de las OSC mexicanas están altamente profesionalizadas y contratan personal remunerado, pero muchas se basan sólo en el trabajo voluntario. En general su trabajo es participativo (aunque algunas no lo especifican) y vinculado a las comunidades, ejidos, cooperativas, OSC locales y grupos indígenas.

Su actividad se financia mayoritariamente con recursos internacionales y nacionales, públicos y privados, aunque algunas también reciben cuotas de afiliación de sus socios. Aquellas que reciben recursos gubernamentales lo hacen como retribución a sus actividades de colaboración a través de asesorías y desarrollo conjunto de proyectos. Aproximadamente la mitad de ellas se vincula con los tres niveles de gobierno (federal, estatal y municipal), pero algunas sólo mencionan el nivel federal (Espacios Naturales y Desarrollos Sustentables y Fondo Mexicano para la Conservación de la Naturaleza) y otras sólo el local (ICLEI). Lamentablemente no fue posible obtener información sobre los montos de recursos (financieros y humanos) que manejan.

La mayoría menciona también mecanismos de sistematización y difusión de los conocimientos que van generando: informes, guías, manuales, libros, revistas, artículos de divulgación, páginas web, comunicados de prensa, redes sociales, participación en congresos, etc. Tres de las organizaciones analizadas (Naturalia, Asociación Nacional de Energía Solar e Institute for Transportation \& Development Policy) son responsables de la publicación de revistas especializadas como "Especies. Revista sobre Conservación y Biodiversidad", "Revista de energías renovables" y "Sustainable Transport Magazine", respectivamente.

En opinión de algunos coordinadores 6 las principales contribuciones al DS de sus OSC incluyen:

- Establecimiento y manejo de ANP, restauración de arroyos y cuidado del agua, y freno al avance de la tecnificación e intensificación de la ganadería, que provoca la persecución y muerte de grandes carnívoros, con énfasis sobre el jaguar (Biocenosis).

- Trabajos de conservación y restauración ambiental en el Parque Nacional Desierto de los Leones (Ciudad de México), reforestación y limpieza de bosques, promoción de la participación social y el autoempleo sustentables (Earthgonomic México AC).

- Restauración ecológica del territorio insular mexicano (57 erradicaciones de mamíferos invasores en 37 islas de todos los mares de México, generación de 62 empleos directos de especialistas técnicos y científicos y 400 indirectos, investigaciones científicas en paralelo a las actividades de conservación y restauración, publicaciones, etc.), elaboración participativa y en colaboración con el gobierno federal de la Estrategia Nacional para la Conservación y el Desarrollo Sustentable, y estudios técnicos justificativos de la creación de las Reservas de la Biosfera Isla Guadalupe e Islas del Pacífico de Baja California, también en colaboración con el gobierno federal y con el respaldo de las cooperativas pesqueras (Grupo de Ecología y Conservación de Islas A.C.).

6.- Los que respondieron a la consulta solicitada en agosto de 2016. 


\section{EL DESARROLLO DE LAS ONG DE MÉXICO Y SU COINCIDENCIA CON LOS OBJETIVOS PARA EL DESARROLLO SOSTENIBLE DE LAS NACIONES UNIDAS}

- Programa de Conservación de Tierras mediante el fortalecimiento de ejidos y comunidades en las Sierras La Giganta y Guadalupe (desde hace más de 10 años), impulso junto con los ciudadanos del proceso para decretar a Balandra como Área de Protección de Flora y Fauna (desde 2006), promoción de la primera red de zonas de refugios pesqueros en el país (en 2012), e impulso a la toma de conciencia de la población sobre la necesidad de aprovechar mejor el agua de lluvia en la ciudad de La Paz y la instauración de infraestructura verde que permita la recarga del acuífero (Sociedad de Historia Natural Niparajá).

- Implementación de estrategias de desarrollo sostenible en comunidades campesinas e indígenas generando fuentes de empleo y conservación de su ecosistema (viveros comunitarios, ecoturismo, proyectos productivos con materiales locales, etc.), apropiación de técnicas y estrategias de consumo de energía y construcción (estufas ahorradoras de leña, ecotécnicas de construcción, captación de agua de lluvia, construcción con pacas, etc.), y recuperación de bosques generando empleo temporal en las comunidades campesinas (Naturalia A.C.).

- Impuso de la gobernanza participativa para la efectiva administración de los recursos marinos: toma de decisión colectiva, restitución de derechos y responsabilidades, coproducción del conocimiento, importante contribución de ONG en tres procesos de gestión: el desarrollo del plan de gestión de la pesca del cangrejo, el establecimiento del refugio de Punta San Cosme al corredor Punta Coyote y la implementación de nuevas estrategias para el manejo de la pesca de la corvina del Golfo. También se propone un esquema para evaluar los resultados de estos procesos y la contribución de los diferentes actores vinculados (COBI).

- Defensa legal del Parque Nacional Cabo Pulmo, conservación/protección de Terrenos Nacionales en Laguna San Ignacio y herramientas legales para garantizar la participación pública en procesos de toma de decisión (DAN).

Esta breve síntesis permite reconocer la diversidad de asuntos manejados por las OSC seleccionadas.

\subsection{Actividades desarrolladas por las OSC y Agenda 2030}

Como una primera aproximación a la contribución actual o potencial de las OSC a la Agenda 2030, y a partir de las actividades presentadas en el apartado anterior, el Cuadro No. 2 muestra su nivel de coincidencia con los 17 objetivos mencionados.

El desarrollo de comunidades sostenibles resulta el tema más cubierto, seguido de la protección a los ecosistemas terrestres, la producción y consumo responsable, la educación ambiental y la energía no contaminante. Obviamente, este conjunto de OSC ligadas a la actividad No. 12 de DS no alcanza a cubrir todos los objetivos propuestos para el 2030, sino que requiere complementarse en materia de pobreza, reducción de las desigualdades, educación de calidad en sentido amplio (no sólo ambiental), trabajo decente y crecimiento económico; industria, innovación e infraestructura; paz, justicia e instituciones sólidas; y alianzas para lograr los objetivos. Algunos de ellos se cubren en otras de las 19 
actividades previstas por la LFFAROSC como las relativas a asistencia social, educación, apoyo a la alimentación y a la economía familiar, pero siguen sin aparente cobertura algunos temas sociales, económicos y políticos como la desigualdad (no expresamente incluida), el desarrollo industrial y tecnológico, y la seguridad, justicia y solidez institucional.

\section{Conclusiones}

El número de ONG mexicanas ha tenido un rápido crecimiento y se ha concentrado en materia de la actividad 12 de la LFFAROSC, especialmente vinculada al DS. Las regiones Sur y Centro presentan mayor número y densidad, en oposición a las del Norte y Occidente.

Las mismas muestran una antigüedad considerable y una amplia diversidad de temas y regiones cubiertas, que complementan las acciones gubernamentales en materia de DS, principalmente en temas del desarrollo de comunidades sostenibles, la protección a los ecosistemas terrestres, la producción y consumo responsable, la educación ambiental y la energía no contaminante.

A pesar de lo anterior, se identifican algunos ODS donde las OSC revisadas no manifiestan expresamente actividad: pobreza, reducción de las desigualdades, hambre cero, educación de calidad en sentido amplio (no sólo ambiental), entre otras. Sin embargo, éstos pueden estar cubiertos por ONG que desempeñan otras actividades de la LFFAROSC. Esto puede deberse a que desde sus inicios el DS se ha administrado desde el eje ambiental, en el caso de México por la Semarnat, sin embargo las componentes social y económica son responsabilidad de otros sectores.

En cambio, existen ODS no contemplados en la LFFAROSC, en particular los más vinculados a cuestiones económicas y políticas en las que las OSC tienen menos posibilidades de incidir en función de sus recursos, razón por la que la responsabilidad queda en manos casi exclusiva del gobierno: trabajo decente y crecimiento económico, desarrollo industrial y tecnológico, y seguridad, justicia y solidez institucional.

Como se menciona en la Agenda 2030, los ODS son de carácter integrado e indivisible, es decir, tomar acción sobre uno de ellos tiene consecuencias sobre los otros y éstas pueden ser positivas 0 negativas. De tal manera que deben ser evaluados en conjunto como un sistema complejo.

En general, existe una resistencia por parte de las OSC a ser evaluadas probablemente debido a la falta de cultura en evaluación y al miedo a ser penalizadas. Las organizaciones prefieren invertir en más acciones, antes de hacerlo en evaluaciones para conocer el impacto de su trabajo. Sólo hacen reportes o informes de resultados o metas alcanzadas. 


\section{EL DESARROLLO DE LAS ONG DE MÉXICO Y SU COINCIDENCIA CON LOS OBJETIVOS PARA EL DESARROLLO SOSTENIBLE DE LAS NACIONES UNIDAS}

En síntesis, existe un número importante de objetivos de la Agenda 2030 en que las OSC mexicanas manifiestan tener actividad, la que ya está contribuyendo a su logro 0 , al menos, tiene el propósito de hacerlo. En consecuencia, su participación es relevante en el contexto nacional y fundamental en algunas regiones del país con menor presencia de programas gubernamentales.

\section{Cuadro 2. Coincidencia de las actividades de las OSC con los Objetivos para el Desarrollo Sostenible}

\begin{tabular}{|c|c|c|c|c|c|c|c|}
\hline \multirow{2}{*}{$\begin{array}{l}\text { OSC } \\
\text { estudiadas }\end{array}$} & \multicolumn{7}{|c|}{ Objetivos de la Agenda 2030} \\
\hline & $\begin{array}{l}\text { Educación } \\
\text { ambiental }\end{array}$ & \begin{tabular}{l}
\multicolumn{1}{c}{ Energía } \\
asequible y no \\
contaminante
\end{tabular} & $\begin{array}{c}\text { Ciudades y } \\
\text { comunidades } \\
\text { sostenibles }\end{array}$ & $\begin{array}{l}\text { Producción } \\
\text { y consumo } \\
\text { responsables }\end{array}$ & $\begin{array}{l}\text { Acción por } \\
\text { el clima }\end{array}$ & $\begin{array}{c}\text { Ecosistemas } \\
\text { terrestres }\end{array}$ & Otros \\
\hline Centro Mexicano de Derecho Ambiental (CEMDA) & & & & & & & Justicia e instituciones \\
\hline Pronatura México & & & $x$ & & & $x$ & \\
\hline Espacios Naturales y Desarrollo Sustentable & $x$ & & $x$ & & & $x$ & \\
\hline Fondo Mexicano para la Conservación de la Naturaleza & & & $x$ & & & $x$ & Alianzas; Vida submarina \\
\hline Beta Diversidad & & $x$ & $x$ & & & $x$ & \\
\hline Naturalia & $x$ & & $x$ & & & $x$ & \\
\hline World Wildlife Fund (WWF) México & & & $x$ & & & & \\
\hline Conservación internacional & $x$ & & $x$ & $x$ & $x$ & $x$ & $\begin{array}{l}\text { Salud y bienestar; } \\
\text { Agua limpia; Hambre cero }\end{array}$ \\
\hline Greenpeace México & & $x$ & & $x$ & $x$ & $x$ & Vida submarina \\
\hline Comunidad y Biodiversidad & & & $x$ & $x$ & & & Vida submarina \\
\hline The Nature Conservancy Mexico & & & & $x$ & $x$ & $x$ & \\
\hline Consejo Civil Mexicano para la Silvicultura Sostenible & & & & $x$ & & & \\
\hline Insititute for Transportation \& Development Policy & & & $x$ & $x$ & & & $\begin{array}{l}\text { Industria, innovación, } \\
\text { infraestructura }\end{array}$ \\
\hline \begin{tabular}{|l|} 
Acción Cultural Madre Tierra, A.C. e Iniciativa \\
Mexicana de Aprendizaje para la Conservación
\end{tabular} & & & $x$ & & & & Igualdad de género \\
\hline |CLEI-Gobiernos Locales por la Sustentabilidad & & $x$ & $x$ & & $x$ & & $\begin{array}{l}\text { Agua limpia, Industria, } \\
\text { innovación, infraestructura }\end{array}$ \\
\hline Biocenosis A.C & & & $x$ & & & $x$ & \\
\hline Grupo de Ecología y Conservación de Islas & $x$ & & & & & $x$ & \\
\hline Grupo de Estudios Ambientales AC & & $x$ & & $x$ & $x$ & & \\
\hline Asociación Nacional de Energía Solar & $x$ & $x$ & & & & & \\
\hline Fondo para la Comunicación y la Educación Ambiental AC & $x$ & & & & & & \\
\hline Estudios Rurales y Asesoría Campesina AC & & & & $x$ & & & \\
\hline Total & 6 & 5 & 12 & 8 & 5 & 10 & \\
\hline
\end{tabular}

FUENTE: Elaboración propia. 


\section{Aportes, limitaciones y perspectivas}

Las 21 OSC estudiadas en el marco de la actividad No.12 de la LFFAROSC fueron seleccionadas por contar con el reconocimiento gubernamental, de otras OSC y de la comunidad académica por su contribución al DS y por disponer de información. Sin embargo, no son representativas del universo de más de 5,000 al que pertenecen.

Las variables consideradas en el análisis realizado resultaron pertinentes para el objetivo del trabajo, especialmente la descripción de las actividades de las OSC, su antigüedad y experiencia, su relación con el gobierno y la sociedad civil, así como el origen de sus recursos. Sin embargo, hubiera sido deseable disponer de datos sobre los recursos financieros y humanos (remunerados o voluntarios) que disponen.

La información utilizada para caracterizar a las OSC proviene fundamentalmente de sus propias páginas web y de las consultas directas realizadas cuando los datos recabados no aparecen en los registros oficiales. Su verificación empírica ameritaría un estudio profundo de cada caso que, si bien ya se ha iniciado, no fue factible en esta primera aproximación.

En relación con la producción de conocimiento por parte de las OSC, sólo se pudo hacer una descripción general del variado tipo de documentos que utilizan para difusión, pero se requeriría un análisis profundo de su contenido para poder opinar sobre su calidad y utilidad.

Si bien el estudio realizado muestra sintéticamente el desarrollo de las ONG en México y efectúa una primera aproximación a la contribución que la actividad de las principales de ellas realiza al DS, queda pendiente para nuevas investigaciones que dispongan de los recursos necesarios, hacer una evaluación de impacto integral, que permita identificar, medir, explicar y valorar los efectos provocados por la gestión del conjunto de ONG de cada región del país en torno a los objetivos de la Agenda 2030 de la ONU. 


\section{Referencias}

ACOTTO, L. (2003): Las organizaciones de la sociedad civil: un camino para la construcción de ciudadanía, Buenos Aires: Espacio Editorial.

BRAND, U. \& GORG, Ch. (2000): "Contradicciones sustentables: el papel de las ONGs en la política internacional de la biodiversidad”, Estudios Latinoamericanos, Nueva Época, Año VII, 14, 45-69.

AGUIRRE, A., SAMANIEGO-HERRERA, A., LUNA-MENDOZA, L., ORTIZ-ALCARAZ, A., RODRÍGUEZ-MALAGÓN, M., FÉLIX-LIZÁRRAGA, M., MÉNDEZ-SÁNCHEZ, F., GONZÁLEZGÓMEZ, R., TORRES-GARCÍA, F., HERNÁNDEZ-MONTOYA, J.C., BARREDO-BARBERENA, J.M. \& LATOFSKI-ROBLES, M. (2011): "Eradications of invasive mammals on islands in Mexico: the roles of history and the collaborations between government agencies, local communities and non-government organization", Island invasives: eradication and management. IUCN, Gland, Switzerland, 386-394.

BERMÚDEZ, O. \& LOMBANA, M. (2009): Organismos No Gubernamentales que trabajan en Educación para el Desarrollo Sostenible y en Educación Ambiental en América Latina y el Caribe, Santiago: OREALC-UNESCO.

CAICEDO, S. \& SOLARTE, L. (2015): "Empoderamiento de mujeres de una ONG colombiana. Un estudio de caso simple", Revista de Administração Pública, 49(6).

CANTO, M. (2002): Interacción de las organizaciones de la sociedad civil, Santo Domingo: BID-IntecOxfam.

CASTAÑEDA, E. (2016): Cooperación internacional para el desarrollo local sustentable: prácticas de participación de organizaciones de la sociedad civil en el Corredor Biológico Mesoamericano en Quintana Roo, tesis de Maestría en Cooperación Internacional para el Desarrollo del Instituto José María Luis Mora, México.

CODIGO CIVIL FEDERAL, www.diputados.gob.mx/LeyesBiblio/pdf/2_241213.pdf [13/01/2016].

COMOLET (1994): L'évaluation et la comptabilisation du patrimoine naturel, définitions, méthodes et pratiques, Paris : L'Harmattan.

CONABIO-CONANP-TNC-PRONATURA (2007): Análisis de vacíos y omisiones en conservación de la biodiversidad marina de México: océanos, costas e islas, Comisión Nacional para el Conocimiento y Uso de la Biodiversidad - Comisión Nacional de Áreas Naturales Protegidas - The Nature Conservancy - Programa México - Pronatura A. C. México, DF.

CONCHA CANTÚ, H. (1997): "Las Organizaciones No Gubernamentales”. En: García Ramírez, S., Los valores en el derecho mexicano. Una aproximación, UNAM - Fondo de Cultura Económica, México. 
CONEVAL (2016): Medición de la pobreza, en http://www.coneval.org.mx/Medicion/Paginas/Pobrezalnicio.aspx, [14/12/2017].

DAVIES, R. (2001): "Monitoring and evaluating NGO achievements". En: Potter, R.B y V. Desaid, Arnold companion to development studies. En: www.mande.co.uk/docs/arnold.htm [25 de abril de 2017].

DELER, J.P., FAURÉ, Y.A., PIVETEAU, A. \& ROCA, P.J. (Dir.) (1998): ONG et développment. Société, économie, politique, Paris : Karthala.

DRUCKER, P. (1990): Managing the Non-Profit Organization, New York: Harper Collins.

El Economista, "EI PIB de México creció 2.3\% en 2016", 22 de febrero de 2017.

El Economista, "México cuenta con 123.5 millones de habitantes", 10 de julio de 2017.

ESPINOSA-ROMERO, J., RODRÍGUEZ, L.F., HUDSON, A., VILLANUEVA-AZNAR, C. \& TORRE, J. (2014): "The changing role of NGos in Mexican small-scale fisheries: From environmetal conservation to multi-scale governance", Marine Policy, 50, 290-299.

FERNANDES, R. (1994): Privado aunque público, Brasil: Civicus.

FOWLER, A., GOOLD, L. \& JAMES, R. (1995): Participatory Self-Assessment of NGO capacity, Occasional Papers Series, No. 10, Oxford.

GONZÁLEZ, S. (Coord.) (1999): Las organizaciones no gubernamentales mexicanas y la salud reproductiva, México: El Colegio de México.

HOCKINGS, M., STOLTON, S. \& DUDLEY, N. (2000): Evaluating Effectiveness. A framework for Assessing the management of Protected Areas, IUCN.

HOPKINS, R. (1995): Impact assessment. The experience of Latin American NGO's, Oxfam GB y Novib.

HULME, D. \& EDWARDS, M. (Comps.) (1997): NGOs, states and donors: too close for comfort?, Basingstoke.

HURTADO, L. (1995): Desarrollo desde arriba y desde abajo: información, documentación y comunicación en las ONGs de América Latina, Centro de Estudios Regionales Andinos Bartolomé de las Casas, Cuzco, Perú.

INDESOL, Sistema de Información del Registro Federal de las OSC, www.corresponsabilidad.gob.mx [25/01/2016]

INEGI (s/f), Glosario, en www.beta.inegi.org.mx/app/glosario/default.html?p=csisflm\#letraGlol [03/02/2017]

INSTITUTE FOR TRANSPORTATION \& DEVELOPMENT POLICY (publicación anual), Sustainable Transport Magazine, www.itdp.org/library/sustainable-transport-magazine, [12/06/2016]. 


\section{EL DESARROLLO DE LAS ONG DE MÉXICO Y SU COINCIDENCIA CON LOS OBJETIVOS PARA EL DESARROLLO SOSTENIBLE DE LAS NACIONES UNIDAS}

KRUSE, S. et al. (1997): Searching for impact and methods: NGO evaluation synthesis study, resumen del informe del Grupo de Expertos en Evaluación de la OCDE, www.valt.helsinki,fi/ids/ngo [04/10/2016].

LÉNA, P. (1998): "ONG, développment durable et société en Amazonie brésilienne". En Deler, J.P. (Dir), ONG et développment. Société, économie, politique, Paris : Karthala.

LEVERINGTON (2008): Management effectiveness evaluation in protected areas - $A$ global study. Overview of approaches and methodologies, The Nature Conservancy-WWF-The University of Queensland-IUCN-WCPA-Biodiversity Indicators Partnership.

Ley Federal de Fomento a las Actividades realizadas por las Organizaciones de la Sociedad Civil, www.corresponsabilidad.gob.mx/sistema/sirfosc/seccionpagina/contenido/seccioncontenido/Anexos/archi vos/Anexo80.pdf [25/01/2016]

LÓPEZ, J. \& HINCAPIÉ, S. (2015): "Campos de acción colectiva y las ONG de derechos humanos. Herramientas teóricas para su análisis", Sociológica, 30(85).

LLORENTE-BOUSQUETS, J. \& OCEGUEDA, S. (2008): "Estado del conocimiento de la biota", Capital Natural de México, Vol. I: Conocimiento actual de la biodiversidad. Conabio, México, 283-322.

MÉNDEZ, J.L. (1995): Organizaciones civiles y políticas públicas en México y Centroamérica, M.A. Porrúa y Academia Mexicana de Investigación en Políticas Públicas, México.

MUÑIZ, L. (2016): “Crecimiento económico de México y ajuste en sus expectativas", El Financiero, 13 de agosto, México.

NATURALIA (publicación periódica), Revista Especies sobre Conservación y Biodiversidad, https://www.joomag.com/magazine/especies-revista-sobre-conservaci\%C3\%B3n-y-biodiversidadabril-junio-2015/0979615001434151979?page=25, [23/06/2016]

NEW ECONOMICS FOUNDATION (1998): Towards understanding NGO Works on Policy, Londres: NEF.

OAKLEY, P. (1999): The Danish NGO impact study. A review of Danish NGO activities in developing countries, Oxford: INTRAC.

OLVERA RIVERA, A. (1998): "Problemas conceptuales en el estudio de las organizaciones civiles: de la sociedad civil al tercer sector". En: De lo cívico a lo público: una discusión de las organizaciones civiles, México: Red Mexicana de Investigadores de Organismos Civiles.

OLVERA RIVERA, A. (2002): "Democracia y sociedad civil en México: lecciones y tareas", Revista de Comercio Exterior, 52(5).

ONU (1945): Carta de las Naciones Unidas, www.un.org/es/charter-united-nations/index.html [03/02/2017]

ONU (1987): Informe Brundtland, "Nuestro futuro en común", www.un.org/es/comun/docs/?symbol=A/42/427 [02/12/2015] 
ONU (s/f): Agenda 2030 para el Desarrollo Sostenible, www.un.org/sustainabledevelopment/es/laagenda-de-desarrollo-sostenible [14/02/2016].

POMEROY, R., PARKS, J.E. \& WATSON, L.M. (2006): Cómo evaluar un AMP. Manual de indicadores naturales y sociales para evaluar la efectividad de la gestión en Áreas Marinas Protegidas, IUCN-WWF-NOAA.

POUYLLAN, M. \& SAAVEDRA, A.R. (1998): "États et ONG: acteurs du rapport environnement-société". En: Deler, J.P. (Dir.), ONG et développment. Société, économie, politique, Paris : Karthala.

Registro Federal de las Organizaciones de la Sociedad Civil, http://indesol.gob.mx/osc/registro-osc [15/02/2016].

RIDDELL, R.C., KRUSE, S-E., KYOLLEN, T., OJANPERA, S., VIELAJUS, J-L. (1997): Searching for Impact and Methods: NGO Evaluation Synthesis Study. A Report produced for the OECD/DAC Expert group on Evaluation, Department for International Development Cooperation, Ministry of Foreign Affairs, Helsinki, en www.valt.helsinki.fi/ids/ngo [15 de febrero de 2014].

ROCHE, C. (1999): Impact Assessment for Development Agencies, Gran Bretaña: Oxfam, Adaptado como Avaliaçao de Impacto dos trabalhos de ONGs (2000), Brasil: Cortez Editora, Oxfam y ABONG.

RODRÍGUEZ, L. (2015): "Metodologías de evaluación de la sustentabilidad en Áreas Naturales Protegidas. Estado del arte y propuesta metodológica para México", Studia Politicae, 34, Argentina: Universidad Católica de Córdoba.

SEDESOL/INDESOL (2015): Curso "Profesionalización y capacitación para las organizaciones de la sociedad civil y otros actores sociales", presentación en Power Point.

SEGOB, Directorio de Organizaciones Sociales, www.organizacionessociales.segob.gob.mx/en/Organizaciones_Sociales/Directorio_de_OSC [28/02/2016].

SEGOB, Área de Desarrollo Político, www.desarrollopolitico.gob.mx [23/12/2015].

SEMARNAT (2016): consulta a la Dirección General Adjunta de Participación Ciudadana de la UCPAST.

SERRANO, M. (2002): Las ONG y la política: detalles de una relación, Madrid: Istmo SA.

Sistema de Información del Registro Federal de las OSC, www.corresponsabilidad.gob.mx, [12/03/2016]

THOMPSON, A. et al. (1995): Políticas Públicas y sociedad civil en Argentina: el papel de las organizaciones sin fines de lucro, CEDES, Argentina.

TORRES, B. (1999): "Las ONG ambientales en las relaciones México-Estados Unidos", Foro Internacional, Vol. XXXIX, 4 (158). 